\title{
ARTE RUPESTRE EN PASOS CORDILLERANOS DEL BOSQUE ANDINO PATAGÓNICO (EL MANSO, REGIÓN DE LOS LAGOS Y PROVINCIA DE RIO NEGRO, CHILE-ARGENTINA)
}

\author{
MARÍA MERCEDES PODESTÁ;, CRISTINA BELLELLI", RAFAEL LABARCA"*, \\ ANA M. ALBORNOZ ${ }^{\cdots \cdots}$, ANABELLA VASINI ${ }^{* \cdots \cdots}$ Y ELENA TROPEA ${ }^{* \cdots \cdots}$
}

\begin{abstract}
RESUMEN
Se presenta información referida a los sitios arqueológicos con arte rupestre conocidos hasta el momento en la cuenca del río Manso (Norpatagonia), en territorio argentino, y a dos sitios recientemente relevados ubicados en el valle de dicho río en Chile. Las relaciones estilísticas entre los sitios de ambos sectores de la Cordillera de los Andes permiten incluirlos dentro de una misma unidad cultural y temporal, relacionada con el estilo de Grecas o TAGC y también con la modalidad del Ámbito Boscoso Lacustre del Nordeste de Patagonia propia de los cazadores recolectores del Holoceno tardío en Patagonia.
\end{abstract} recolectores.

PALABRAS CLAVE: Arte rupestre - Bosque andino-patagónico - arqueología de cazadores ROCK ART IN THE MOUNTAIN PASSES OF THE ANDEAN-PATAGONIC FOREST
(THE MANSO, LAKES REGION AND PROVINCE OF RÍO NEGRO, CHILE-ARGENTINA)

\section{ABSTRACT}

This paper presents information related to archaeological sites with rock art known so far in the basin of Manso river (northern Patagonia), in Argentine territory, and two sites recently discovered in the valley of the same river in Chile. The stylistic relations between all the sites located en both sides of the Cordillera de los Andes permits their inclusion in the same cultural and temporal unit related to the Grecas style o TAGC, and specially the Ámbito Boscoso Lacustre modality of the hunther gatherers's of the late Holocene in Patagonia.

KEY WORDS: Rock art - Patagonic Andean forest - Hunter gatherers archaeology.

\footnotetext{
Instituto Nacional de Antropología y Pensamiento Latinoamericano (INAPL) - 3 de Febrero 1370 - 1426 Buenos Aires - Argentina. mercedespodesta@yahoo.com.

* Consejo Nacional de Investigaciones Científicas y Técnicas (CONICET) - Instituto Nacional de Antropología y Pensamiento Latinoamericano (INAPL) - Universidad de Buenos Aires (UBA), Argentina - bellelli@mail.retina.ar.

** Programa de doctorado del la Universidad del Centro de la Provincia de Buenos Aires (UNCPBA), Argentina - R.labarca.e@gmail.com.

*s** Dirección General de Cultura de la Provincia de Río Negro, Argentina - anaalbornoz@bariloche.com.ar.

*sss Instituto Nacional de Antropología y Pensamiento Latinoamericano (INAPL), Argentina - avasinia@yahoo.com.ar.

***s* Instituto Nacional de Antropología y Pensamiento Latinoamericano (INAPL), Argentina - tellotro@yahoo.com.
} 


\section{INTRODUCCIÓN Y AMBIENTE}

Las investigaciones arqueológicas que se focalizan en el ámbito boscoso de la cuenca del río Manso inferior ${ }^{1}$ (SO de la provincia de Río Negro, Argentina) desde 2003, pero más intensamente desde 2005, permiten ir acrecentando la información acerca del uso de los ambientes de bosque por parte de los cazadores-recolectores en este sector del Norte de la Patagonia durante el Holoceno tardío (Bellelli et al. 2007, 2008; Podestá y Albornoz 2007; Podestá et al. 2008)² (Fig. 1). Estos trabajos continúan y complementan otros emprendidos en 1995 en ambientes de bosque y su transición hacia la estepa patagónica (ecotono), localizados al Noroeste de la provincia del Chubut, específicamente en la Comarca Andina del Paralelo $42^{\circ}$ (en adelante CA $\left.42^{\circ}\right)$, región que se inicia inmediatamente al Sur

1 El Manso recibe distintas acepciones de uno y otro lado de la frontera. Como se indicó, el último tramo del río en territorio argentino se denomina Manso inferior pues existe un sector, aguas arriba y fuera del ámbito de esta investigación, que se llama Manso superior. Se trata más de un topónimo que de una sectorización del cauce fluvial. En Chile, el mismo río, a pesar de encontrarse aguas abajo del Manso inferior, se denomina Manso a secas.

2 Los trabajos son financiados por la Agencia de Promoción de la Ciencia y la Tecnología, el Consejo de Investigaciones Científicas y Técnicas, la Universidad de Buenos Aires y el Instituto Nacional de Antropología y Pensamiento Latinoamericano, Secretaría de Cultura, Argentina. del valle del río Manso inferior hasta la localidad de Cholila y el Parque Nacional Los Alerces (Bellelli et al. 2003; Carballido Calatayud 2007; Fernández 2006, 2007; Podestá et al. 2000, 2007; Scheinsohn y Matteucci 2004, 2005).

El río Manso inferior (nombre que tiene exclusivamente en el sector argentino, como se aclaró en la Nota 1) corre en dirección Este-Oeste a lo largo de $44 \mathrm{~km}$ y recibe a lo largo de su recorrido algunos afluentes entre los que se destacan los ríos Villegas y Foyel. Estos cursos de agua surcan valles relativamente angostos encerrados por cerros de mediana altura cuyas laderas presentan vegetación de bosque caducifolio. Existen distintas especies de Nothofagus aunque también está presente Austrocedrus chilensis en grandes sectores de los faldeos. Luego de trasponer las bajas alturas características de la Cordillera de los Andes en estas latitudes (aproximadamente $41^{\circ} 30^{\prime}$ lat. sur), atraviesa el límite internacional (Paso El León o Manso). A pocos kilómetros de ese punto el río cambia de rumbo abruptamente hacia el Sur y desciende por un terreno de gradiente muy pronunciada que lo encauza en profundos cañadones atravesando así el territorio chileno hasta que vierte sus aguas en el río Puelo y posteriormente en el Océano Pacífico (Fig. 1). Con respecto a la vegetación, a medida que se produce el avance hacia la cordillera y al límite argentino-chileno, ésta se hace más densa ya que comienzan a aparecer especies típicas de

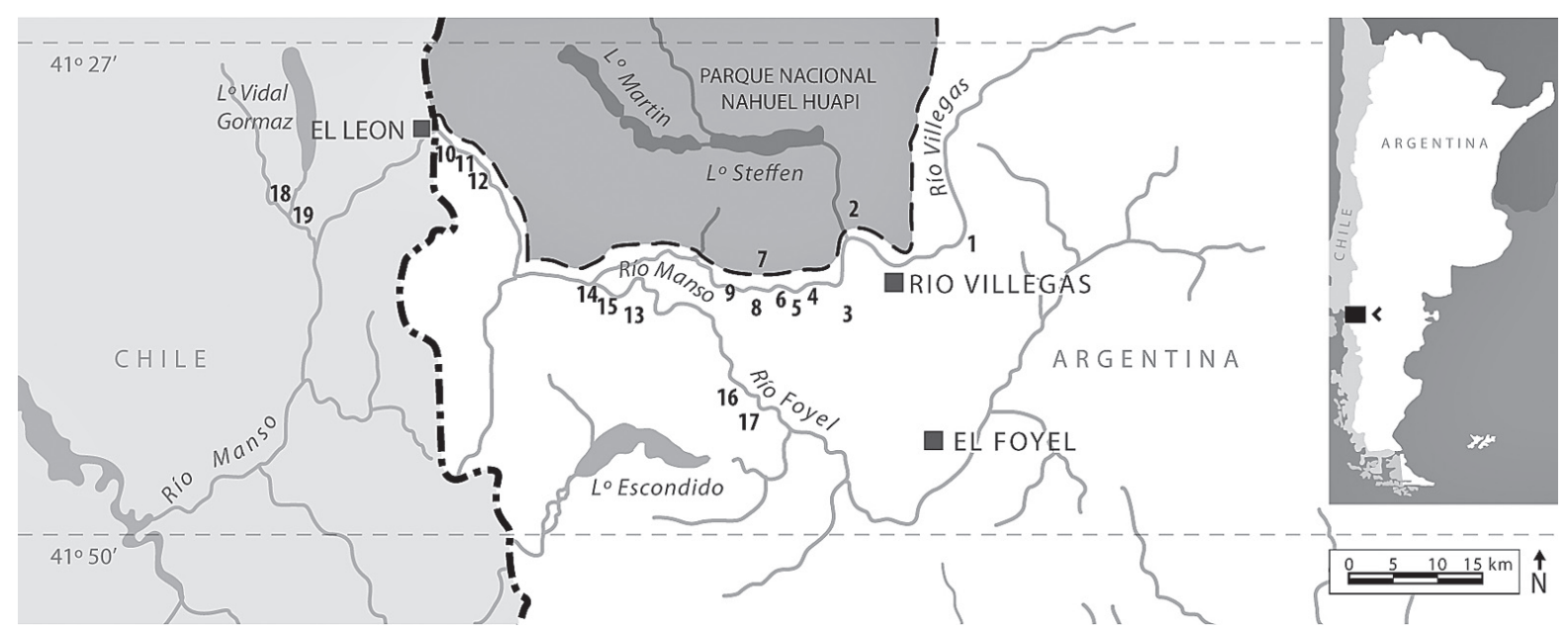

Fig. 1. Mapa de la región con la ubicación de los sitios conocidos hasta el momento: 1.- Seccional Villegas. 2.- Piedra Huenchupán. 3.- Población Anticura. 4.- Paredón Lanfré. 5.- Bloque Lanfré. 6.- Campamento Argentino. 7.- Belmar. 8.- Casa de Piedra (Carro). 9.- Andrade. 10.- Santa Lucía 1. 11.- Santa Lucía 2. 12.- Santa Lucía 3. 13.- Peumayén 1. 14.- Peumayén 2. 5.- Puente Verde. 16.- Mallín Redondo. 17.- Río Foyel 1. 18.- Torrentoso 01. 19.- Correntoso 01. 
la Selva Valdiviana. El rico sotobosque hace que la circulación humana, a medida que se avanza hacia el Occidente, sea cada vez más complicada.

A pesar de la dificultad que adquiere el tránsito por el corredor que define esta cuenca, la región Manso inferior-Foyel-Villegas-Manso ofrece la posibilidad de circulación en la zona cordillerana, conectando ambientes ecotonales y esteparios del Este con la costa pacífica, en el Oeste. En tiempos históricos este paso era utilizado para los arreos de vacunos hacia la localidad chilena de Cochamó, por lo que se lo conocía como Paso de Cochamó (Bellelli et al. 2007). Actualmente del lado argentino existe un camino que se interrumpe en el límite binacional mientras que en el sector chileno se está proyectando uno ya que la circulación es, por el momento, exclusivamente pedestre o a caballo.

Prospecciones realizadas recientemente por uno de los autores de este trabajo en el Manso (Labarca 2007), dentro de territorio chileno, permiten sugerir que, al igual que lo observado históricamente, la Cordillera de los Andes no constituyó una frontera infranqueable para los grupos humanos que habitaron antiguamente el área ${ }^{3}$. Se postula que éstos se habrían desplazado regularmente por la región, aprovechando las bajas alturas y la existencia de vías naturales de circulación como son los valles de los ríos, integrándola dentro de los amplios circuitos de movilidad que antiguamente estos cazadores desplegaron. Esta idea general surge a partir de la existencia, en ambos sectores cordilleranos, de sitios con arte rupestre pintado que comparten una misma tendencia estilística. Con este breve informe se ofrece al lector una síntesis de la información obtenida hasta el momento relacionada principalmente con la actividad de producción de pinturas rupestres en la región. Es de esperar que con el avance de las investigaciones en ambos sectores (chileno-argentino) sea posible entregar en el futuro

3 En estas latitudes la Cordillera Patagónica Septentrional, como se denomina en Argentina al sector norte de los Andes patagónicos entre los $37^{\circ} \mathrm{S}$ y los $45^{\circ} \mathrm{S}$ (latitud a partir de la cual se desarrolla casi totalmente en territorio chileno), presenta cordones montañosos que "... alcanzan alturas variables generalmente comprendidas entre los 2000 y 2500 msnm. Las mayores elevaciones se encuentran sobre el límite internacional, destacando /.../ el cerro Tronador (3500 )/.... Las alturas tienden a descender hacia el sur de la unidad..." (Pereyra 2003:111). nuevas evidencias arqueológicas que abonen esta idea preliminar referida a la interacción entre uno y otro lado de la cordillera.

En el área en su conjunto (región de Cholila, valle del río Epuyén en la $\mathrm{CA} 42^{\circ}$ y cuenca del río Manso inferior), específicamente a partir de los 2.000 años AP, se constata la ocupación humana ininterrumpida de estos ambientes boscosos, si bien tanto al Norte como al Sur del área existen ocupaciones fechadas en momentos más tempranos. Una explicación que se sugirió para justificar estas ocupaciones del bosque es que los cazadoresrecolectores explotaron este ambiente para obtener recursos vegetales específicos y exclusivos de ese ecosistema como plantas medicinales y tintóreas, resinas y determinados tipos de maderas y cañas, además de hongos (Bellelli et al. 2003; Gómez Otero y Bellelli 2006). El consumo de huemul (Hippocamelus bisulcus), que se verifica en los sitios de bosque (junto con el de guanaco, Lama guanicoe), sería ocasional y no justificaría la existencia de una estrategia que apuntara solamente a su obtención (Fernández 2006, 2008).

\section{EL ORIENTE ANDINO Y LA CUENCA DEL MANSO INFERIOR}

La identificación de sitios arqueológicos en este sector de la cuenca se dificulta por problemas de visibilidad debido a las características topográficas y de la vegetación de estos ambientes boscosos. A esto se suma la práctica de recolección selectiva de artefactos por parte de aficionados y la existencia de procesos naturales y antrópicos que atentan contra la preservación de algunos vestigios (Bellelli et al. 2008). La mayor visibilidad que presentan los soportes con arte rupestre con respecto a otro tipo de sitios hizo que sólo localizáramos sitios con este tipo de expresiones, además todos ellos fueron indicados por pobladores actuales. Estos mismos pobladores también señalaron la existencia de sectores en la planicie aluvial donde, removiendo la tierra para realizar tareas agrícolas, recuperaron principalmente instrumentos de molienda y tiestos. Estos hallazgos indican la existencia de sitios subsuperficiales tanto en territorio chileno como argentino.

A lo largo de la cuenca del Manso inferior se documentaron 17 sitios con pinturas rupestres de los cuales cinco de ellos fueron excavados o sondeados 
TABLA 1. Sitios con arte rupestre del río Manso inferior y sus afluentes ríos Foyel y Villegas (Argentina) y del río Manso (Chile). Alturas tomadas con posicionador satelital, excepto las que se indican con ca., cuya fuente fue la Hoja IGM 4174-28 (1982).

\begin{tabular}{|c|c|c|c|c|}
\hline SITIO & $\begin{array}{c}\text { CUENCA/ } \\
\text { SUBCUENCA }\end{array}$ & $\begin{array}{l}\text { LOCALIZACIÓN } \\
\text { TOPOGRÁFICA }\end{array}$ & $\begin{array}{l}\text { ALTURA } \\
\text { snm }(m)^{i}\end{array}$ & TIPO DE SITIO \\
\hline 1.- Seccional Villegas & Manso inf./Villegas & Ladera-sector alto & 762 & Pared de afloramiento \\
\hline 2.- Piedra Huenchupán & Manso inf. & Ladera-sector alto & 704 & Bloque \\
\hline 3.- Población Anticura & Manso inf. & Ladera-sector medio & ca. 550 & Alero \\
\hline 4.- Paredón Lanfré & Manso inf. & Pedemonte-llanura aluvial & 489 & Alero y pared \\
\hline 5.- Bloque Lanfré & Manso inf. & Pedemonte-llanura aluvial & 488 & Bloque \\
\hline $\begin{array}{l}\text { 6.- Campamento } \\
\text { Argentino* }\end{array}$ & Manso inf. & Pedemonte-llanura aluvial & 478 & Alero \\
\hline 7.- Belmar & Manso inf. & Llanura aluvial, en costa de río & 496 & Pared de afloramiento \\
\hline $\begin{array}{l}\text { 8.- Casa de Piedra' } \\
\text { (Carro) }\end{array}$ & Manso inf. & Ladera-sector medio & 567 & Cueva pequeña \\
\hline 9.- Andrade & Manso inf. & Ladera-sector medio & ca. 600 & Pared de afloramiento \\
\hline 10.- Santa Lucía 1 & Manso inf. & Ladera-sector alto & 720 & Alero \\
\hline 11.- Santa Lucía 2 & Manso inf. & Ladera-sector medio & 566 & Pared de afloramiento \\
\hline 12.- Santa Lucía 3 & Manso inf. & Ladera-sector medio & 640 & Alero \\
\hline 13.- Peumayén $1^{*}$ & Manso inf. /Foyel & Ladera-sector alto & 857 & Alero y cueva pequeña \\
\hline 14.- Peumayén 2 & Manso inf. /Foyel & Ladera-sector bajo & 510 & Alero y pared \\
\hline 15.- Puente Verde & Manso inf. /Foyel & Ladera-sector bajo & 500 & Pared de afloramiento \\
\hline 16.- Mallín Redondo & Manso inf. /Foyel & Pedemonte-llanura aluvial & 511 & Pared de afloramiento \\
\hline 17.- Río Foyel 1 & Manso inf./Foyel & Sobre curso del río & 478 & Pared de afloramiento \\
\hline 18.- Torrentoso 01 & Manso (Chile) /Torrentoso & Ladera-sector medio & 531 & Pared de afloramiento \\
\hline 19.- Correntoso 01 & Manso (Chile) /Correntoso & Ladera-sector medio & 467 & Pared de afloramiento \\
\hline
\end{tabular}

* Sitios sondeados o excavados.

(Fig. 1). En la Tabla 1 se presenta un listado de los mismos y una somera descripción a través de la cual se consigna la localización topográfica (pedemonte) llanura aluvial, ladera baja, media o alta, costa de río y sobre el río ${ }^{4}$ de cada uno de ellos así como la altura sobre el nivel del mar y el tipo de sitio (cueva pequeña, pared de afloramiento, bloque, alero, alero y pared, alero y cueva pequeña).

Los sitios se distancian entre $0 \mathrm{~m}$ (sitio Río Foyel 1) y $1,5 \mathrm{~km}$ de los ríos. Cuatro de ellos se disponen sobre formaciones rocosas ubicadas en el punto de contacto del pedemonte de los cerros que flanquean los cursos de los ríos y la llanura aluvial que los separa de la costa del río. La mayor parte (once) se distribuye en las laderas bajas, medias y altas de los cerros mientras que sólo dos se encuentran sobre el mismo curso o en costa de río (Fig. 1, 2 y $3)$. Es decir que, considerando las diferencias topográficas que presentan los ambientes circundantes

4 El sitio denominado Río Foyel 1 es la pared de un afloramiento rocoso con pinturas rupestres cuya parte inferior es bañada por las aguas del río homónimo. a los tres cauces de la región (costa de río, llanura aluvial/ pedemonte y ladera baja, media y alta de los cerros), la distribución de los sitios a lo largo de la cuenca del Manso inferior puede considerarse heterogénea.

Los sitios difieren también en cuanto al tamaño y a la cantidad de las pinturas que presentan. Peumayén 2 (P2) y Paredón Lanfré (PL) son los sitios más grandes, el primero tiene 107 motivos y un número mucho mayor de representaciones ${ }^{5}$ que se distribuyen en $68 \mathrm{~m}^{2}$ del soporte rocoso, mientras que en PL se contabilizan 134 motivos dispuestos en $152 \mathrm{~m}^{2}$. Seis de los sitios son pequeños con presencia de uno a cuatro motivos que se yuxtaponen en superficies de entre 1 y $2.5 \mathrm{~m}^{2}$. Los restantes entran en la categoría de sitios medianos con 8 a 32 motivos que ocupan entre 6 y $28 \mathrm{~m}^{2}$ de los soportes rocosos (Podestá y Albornoz 2007; Podestá et al. 2008).

5 Se utilizan las acepciones de motivo y representación según se definen en Aschero y Martel (2002-2003). 


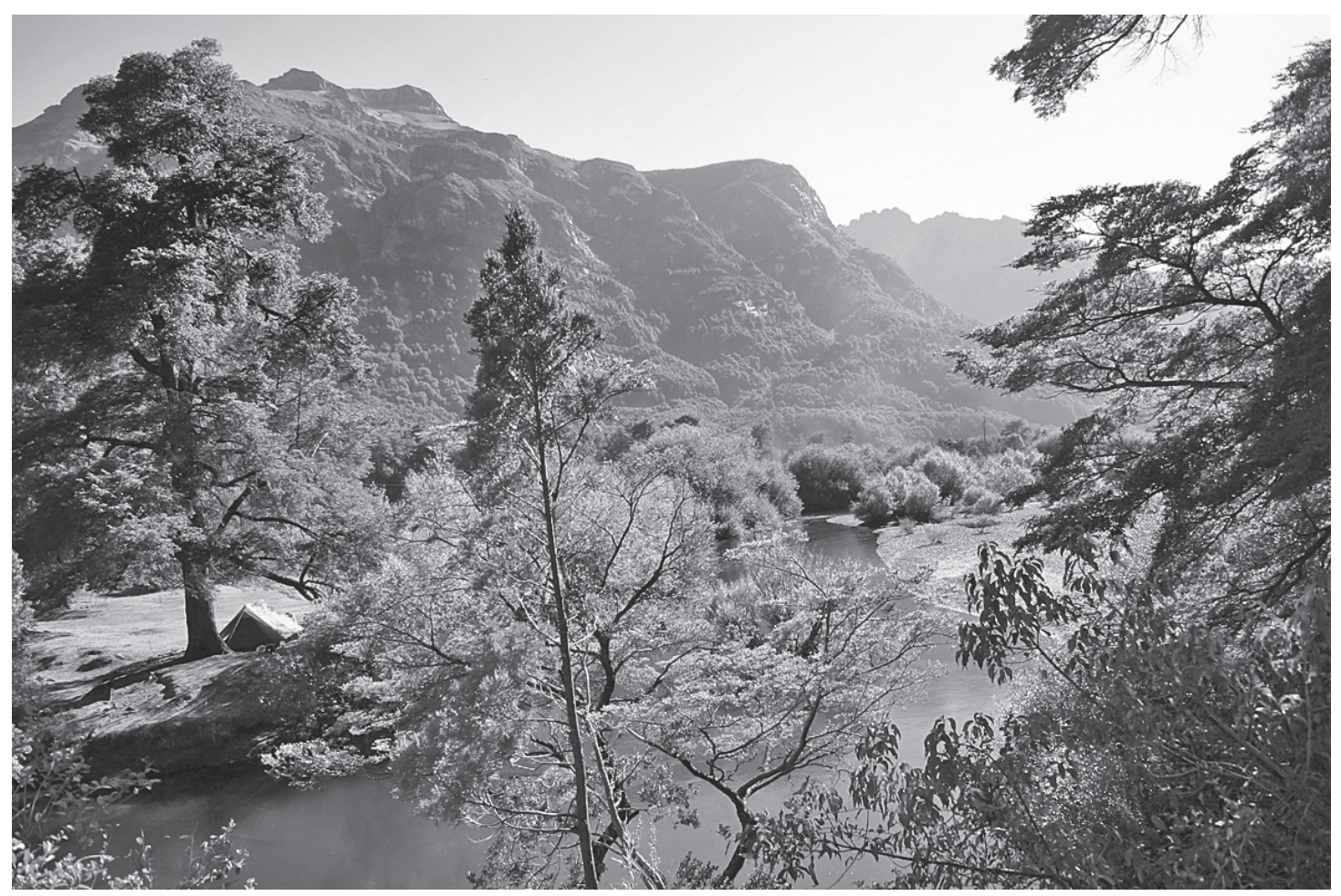

Fig. 2. Vista desde el sitio con pinturas Peumayén 2 hacia el río Foyel, cerca de su desembocadura en el Manso inferior. Argentina.

Los graves problemas de deterioro que afectan a todos los sitios por igual se vislumbran en el alto porcentaje de motivos indeterminados (entre el 20 y $25 \%$ de motivos por sitio). Esto permite sugerir que existe una gran cantidad de pinturas desaparecidos con respecto al repertorio original. Retomando los datos mencionados, la densidad de motivos por superficie varía según los sitios entre 0.50 y 1.66 motivo por $\mathrm{m}^{2}$.

Las abundantes precipitaciones de la región (1.400-1.600 mm anuales y mayores en el sector chileno) producen el lavado y la obliteración de las pinturas a causa de la formación de acreciones de carbonatos y del desarrollo de abundantes microorganismos, con un alto porcentaje de líquenes, sobre la superficie rocosa. El oxalato de calcio presente en las muestras de pinturas de uno de los sitios (PL), identificado a través de la difracción de rayos $\mathrm{X}$, se consideró un posible resultado de las condiciones de tipo climático que afectan a las pinturas (Podestá y Albornoz 2007). En otras muestras se identificó la presencia de sílica amorfa que fue explicada en el análisis de laboratorio como una acreción producida por el agua que afecta a la pared (Helwig 2004). Las características litológicas de gran parte de los soportes rocosos (roca granitoide) también favorecen el deterioro de la superficie que se manifiesta a través de su microexfoliación (Pereyra et al. 2003). En contraste con la información precedente, los daños por acción humana son muy escasos. Salvo contadas excepciones, no existen graffiti, rayados, ni desprendimientos intencionales de la roca, deterioros de origen antrópico comunes de registrar en otras regiones con sitios con arte rupestre ${ }^{6}$.

El arte rupestre de la totalidad de los sitios de la cuenca está asignado a los momentos más tardíos de la secuencia de arte rupestre de Patagonia: la Tendencia Abstracta Geométrica Compleja (TAGC) (s/Gradin 1999). Esta tendencia fue descripta sobre la base de los estilos de Grecas y de Miniaturas definidos previamente por Menghin (1957). El desarrollo de la misma en la Patagonia en su conjunto

6 Las tareas que lleva a cabo el equipo de investigación en todas las escuelas del valle procuran que los pobladores más pequeños se conviertan en los custodios de los sitios arqueológicos. 
se corresponde con un momento avanzado del Holoceno tardío (posterior a los 1.300 años AP). Además de la tendencia mencionada, se reconoce también la presencia de una variedad regional denominada Modalidad del Ámbito Lacustre Boscoso del Noroeste de Patagonia (MALB) (s/Albornoz y Cúneo 2000).

La primera de ellas, la TAGC, se encuentra ampliamente extendida en la vertiente oriental de los Andes, tanto en la zona cordillerana como en la estepa patagónica, llegando inclusive a la costa atlántica y, por el Sur, hasta casi los $48^{\circ}$ de latitud. Esta vasta distribución ha sido explicada por diversos autores a través del crecimiento demográfico y a los sucesivos desplazamientos humanos que se produjeron durante el Holoceno tardío, especialmente durante el momento final en la Patagonia (ver una

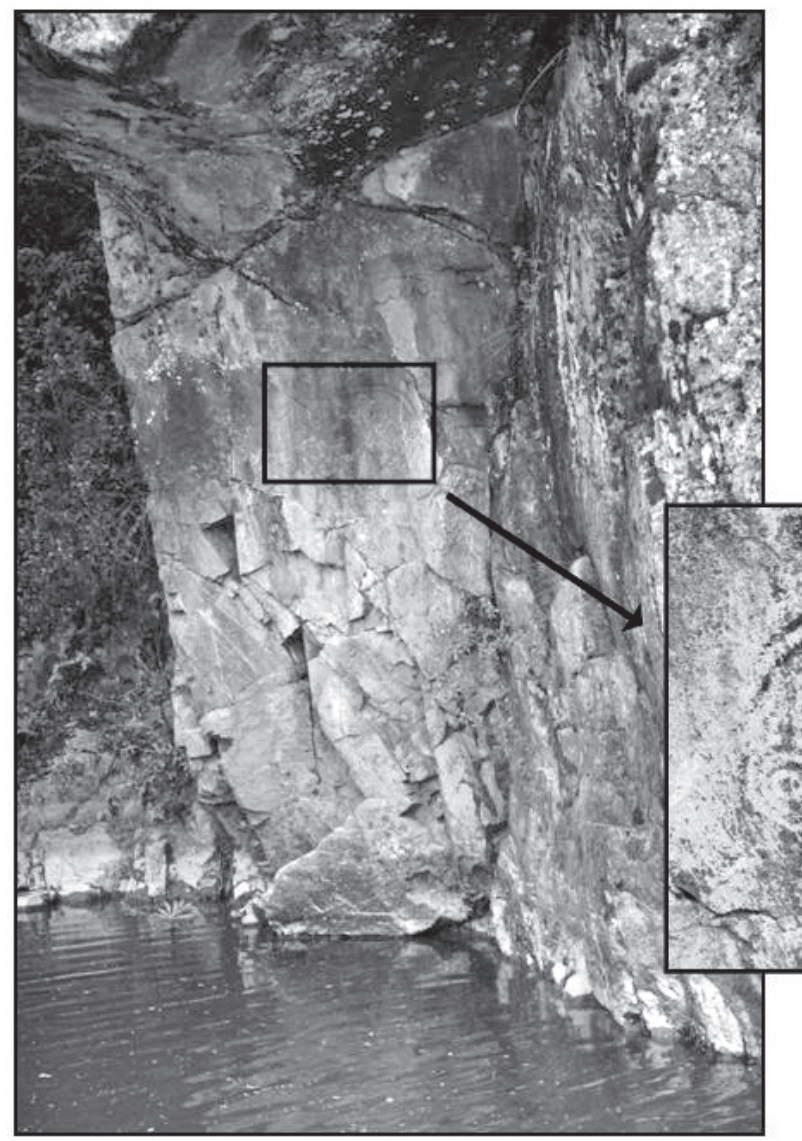

Fig. 3. El río desvió su cauce e inundó al sitio Río

Foyel 1. La ausencia de escala se explica en la imposibilidad de aproximarse a la pared. Las pinturas en rojo y verde se ven en el detalle de la derecha. síntesis reciente en Belardi 2004 y Podestá et al. 2005). En contraste, la modalidad regional (MALB), que se inscribe en la tendencia mencionada, tiene una dispersión espacial más restringida y está concentrada en el corredor de los lagos del bosque andino-patagónico de Patagonia Norte que incluye los lagos Lacar, Nahuel Huapi, Gutiérrez, Mascardi y Guillelmo, ubicados al Norte del área de estudio, en la cuenca del río Manso inferior y en parte de los sitios de la CA42ํㅡㄴ (Albornoz y Cuneo 2000; Podestá y Albornoz 2007; Podestá et al. 2007, 2008).

$\mathrm{El}$ arte rupestre, en su totalidad pinturas, es de tipo abstracto. El patrón formal básico de las representaciones está compuesto por un trazo lineal recto y corto, dispuesto en ángulo recto, que conforma líneas escalonadas o almenadas. También las hay en ángulo agudo que componen líneas en zigzag. Mediante la unión de estos trazos se organizan figuras geométricas simples y complejas. En su conformación más elaborada la línea de patrón escalonado-almenado-zigzag define a las grecas (figura que da nombre al estilo) (Fig. 3). La línea recta sin quebrar se utiliza en general para limitar los enmarcados y para figuras de rombos y tridígitos, entre otros (Fig. 4). Los motivos combinados más comunes son los círculos simples y concéntricos (Fig. $3)$. En este estilo hay una baja incidencia de motivos figurativos (figuras humanas y de animales).

En comparación con la tendencia descripta, la modalidad boscosa (MALB) presenta motivos geométricos más sencillos y menos regulares que los clásicos de la estepa, donde por primera vez se definió el estilo de Grecas o la TAGC. En algunos sitios la representación geométrica se encuentra asociada con figuras de animales (camélido, huemul, caballo) y humanas. Estas últimas suelen presentar tres dedos en sus extremidades, tipo de diseño que puede apreciarse en sitios ubicados en la región de los grandes lagos 


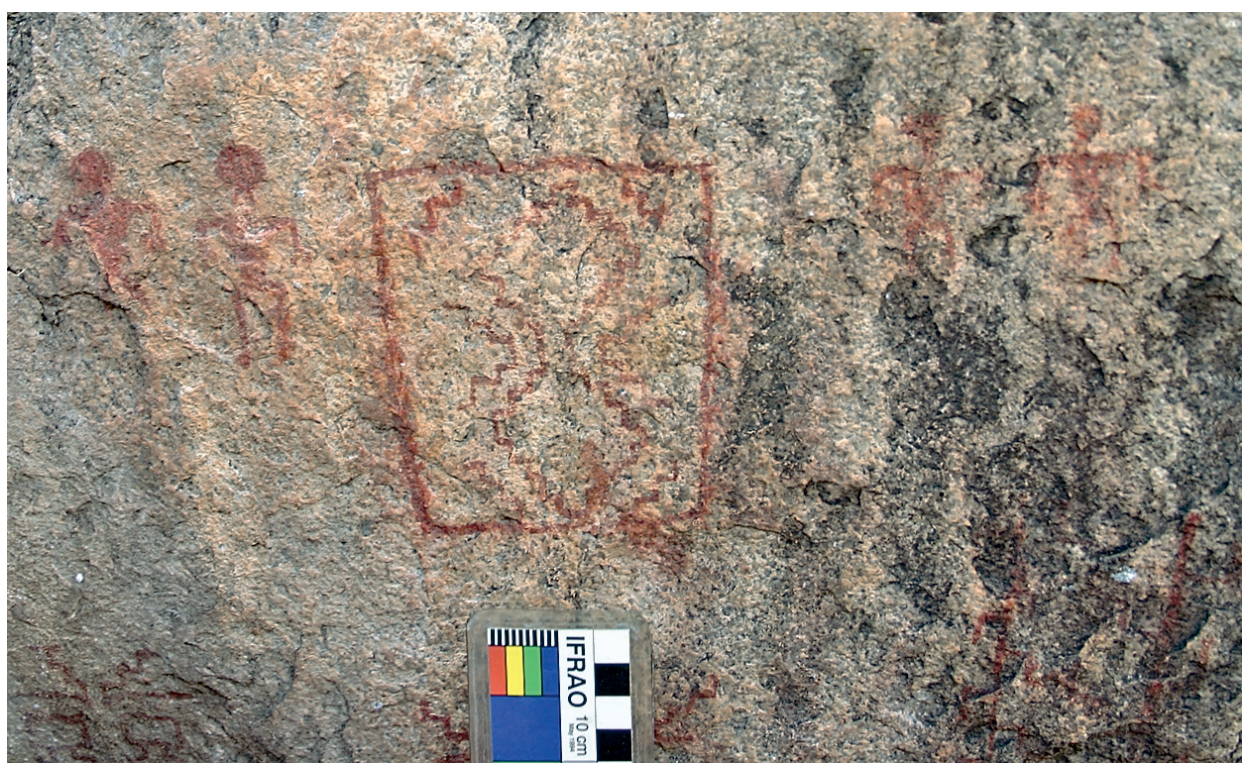

Fig. 4. Representaciones de enmarcados y figuras humanas con indicación de tres dedos, en rojo. Sitio Paredón Lanfré, Argentina.

al Norte de nuestra área de estudio (Puerto Chavol I, Nariz del Diablo I, Cerro Campanario II, El Trébol próximos al Lago Nahuel Huapi; Río Minero I, cercano a Lago Traful; Catritre I en el Lago Lácar) (Albornoz y Cúneo 2000) y en en el sitio Paredón Lanfré (Manso inferior), en el sitio Paredón Lanfré (Podestá y Albornoz 2007) (Fig. 4). También suelen tener indicación de sexo y presentar los brazos en posición elevada hacia ambos lados del cuerpo ("figura orante") (Fig. 5). Las figuras humanas aparecen, por lo general, asociadas con representaciones de cruces, soles, clepsidras y enmarcados, como ocurre en el Paredón Lanfré y otros sitios de la cuenca del Manso inferior, conformando temas que se reiteran (Fig. 4 y 5). Otros sitios documentados presentan solo motivos geométricos como grandes zigzags verticales paralelos a veces realizados a manera de dígitos con arrastre. Dentro de los tipos más destacados están los arcos dobles o cuádruples. Estos sobresalen dentro del conjunto de pinturas del sitio Peumayén 2 por la combinación de colores (dos tonos de rojo, verde y amarillo), su particular morfología y disposición en el soporte, además de su mayor tamaño con respecto a otros motivos. Se trata de largas líneas arqueadas, semejantes al arco iris que, en la mayoría de los casos, se presentan de a pares o, en uno de los casos, en serie de cinco líneas paralelas (Fig. 3 y 5). En la cuenca este motivo aparece también en Paredón Lanfré, Puente Verde y en Río Foyel 1, en territorio argentino, y en el sitio Torrentoso 01, en Chile. En sitios de la CA42으 se lo registra esporádicamente.

El color predominante en las pinturas de la cuenca del Manso es el rojo con diferentes intensidades (rojo oscuro y rojo claro) que se presenta en monocromías (entre un 80 a $85 \%$ de los casos registrados) pero que, además, interviene en bi-

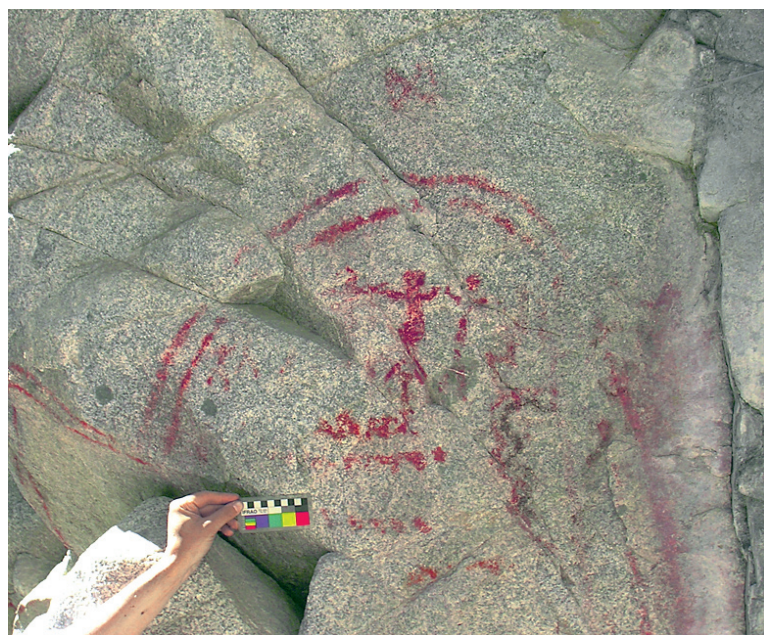

Fig. 5. Arcos y alineación de figuras humanas con los brazos en alto formando un conjunto tonal en rojo. Sitio Peumayén 2. Argentina. 
cromías, combinado con el verde, amarillo rojizo, amarillo o gris oscuro y tricromías, acompañado del verde y amarillo (Fig. 3). En porcentajes menores aparecen el amarillo, naranja y verde. Para conocer la composición mineralógica de las pinturas se cuenta con la información procedente del sitio Paredón Lanfré (Helwig 2004, Podestá y Albornoz 2007). Allí se utilizaron minerales de óxido de hierro como hematita para obtener las distintas tonalidades de rojo. Para el color verde se aprovechó la glauconita o celadonita, denominada también tierra verde. La materia prima utilizada para el amarillo no pudo ser identificada a pesar que se analizaron dos muestras de este mineral. En otros sitios de la Comarca Andina del Paralelo $42^{\circ}$ este tono fue obtenido de la lepidocrocita (polimorfo de la goethita) (Wainwright et al. 2000). El único caso de naranja del sitio pudo haberse obtenido de la combinación de la hematita con caolín como se verificó en una muestra obtenida del sitio Cerro Pintado, próximo a la localidad de Cholila (Sur de la CA42º) (Helwig 2001, Podestá y Tropea 2001, Tropea 2006). Con respecto a los artefactos de aplicación de la pintura sobre el soporte, poco puede decirse. Los finos trazos de algunos motivos en miniatura, que miden entre 2 a $3 \mathrm{~mm}$ de ancho, llevan a suponer el uso de pinceles o hisopos extremadamente delgados, son notables también algunas aplicaciones de pintura con el dedo y el arrastre posterior.

Volviendo a los aspectos cronológicos y considerando nuevamente el área del Manso inferior y la $\mathrm{CA} 42^{\circ}$ en su conjunto, los resultados de las investigaciones alcanzados hasta el momento permiten afirmar que, si bien éste amplio espacio fue ocupado con posterioridad a las 2.000 años AP, la ejecución del arte rupestre fue una práctica que comenzó más tardíamente. Las evidencias obtenidas de la excavación de tres sitios con arte rupestre: Cerro Pintado en Cholila, Risco de Azócar 1 en la cuenca del Epuyén y Paredón Lanfré en el río Manso inferior, permiten sostener que las pinturas más antiguas se habrían producido en algún momento dentro de un lapso comprendido entre los 1.300 y 700 años AP, sin poder precisarlo con mayor exactitud. De Paredón Lanfré y Peumayén 2, los sitios más grandes y con el mayor registro de motivos de toda la cuenca del Manso, surgen evidencias de la existencia de al menos dos o tres momentos de actividad pictórica. En el primero esta diacronía está sustentada en las excavaciones del sitio que permitieron constatar que el mismo estuvo ocupado a lo largo de un período comprendido entre ca. 1.200-300 años AP. Los sitios chicos, por lo contrario, sugieren un único momento de ejecución de pinturas rupestres. Con respecto a las últimas expresiones de esta actividad, la información procedente del sitio Paredón Lanfré, que presenta representaciones de caballos y fechados radiocarbónicos tardíos (330 \pm 50 años AP), indica que la producción de arte rupestre habría superado el siglo XVII (Bellelli et al. 2007; Podestá y Albornoz 2007), es decir que fue contemporánea a los momentos tempranos del contacto hispanoindígena en el área.

\section{EL OCCIDENTE ANDINO Y EL RÍO MANSO}

Las recientes prospecciones de uno de los autores (Labarca 2007) en la cuenca del río Manso, Comuna de Cochamó, Región de los Lagos, Chile, completan la información local. Estos trabajos permitieron el reconocimiento de dos sitios (Torrentoso 01 y Correntoso 01), uno pequeño y el otro mediano (según las categorías descriptas anteriormente), con motivos pintados exclusivamente en rojo y de la misma tendencia estilística que la de los sitios de la cuenca del Manso inferior (sector oriental). Ambos sitios se encuentran enclavados en un ambiente de Selva Valdiviana y se emplazan en laderas altas muy empinadas que encajonan a los ríos Torrentoso y Correntoso, afluentes del Manso.

El primero de los sitios se ubica aproximadamente a unos $400 \mathrm{~m}$ lineales del río Torrentoso, en su ribera este y a unos $100 \mathrm{~m}$ de altura en relación con la caja del río, a media ladera del cerro. Se compone de un único panel con por lo menos 28 motivos reconocibles, abarcando una superficie mínima de 2,6 $\mathrm{m}^{2}$ (Fig. 6). Las representaciones se localizan entre los 0,70 y 1,70 m, de altura en relación al piso actual. Presenta alteraciones naturales y antrópicas. Entre las primeras se incluye el escurrimiento de agua en el sector central que ha deslavado e incluso borrado motivos y algunos líquenes en el sector inferior del panel. Dentro de las alteraciones vandálicas se incluye la realización de graffiti, a través del raspado de la superficie de la roca.

El segundo sitio se localiza en la ribera oeste del río Correntoso, aproximadamente a $1 \mathrm{~km}$ del panel 


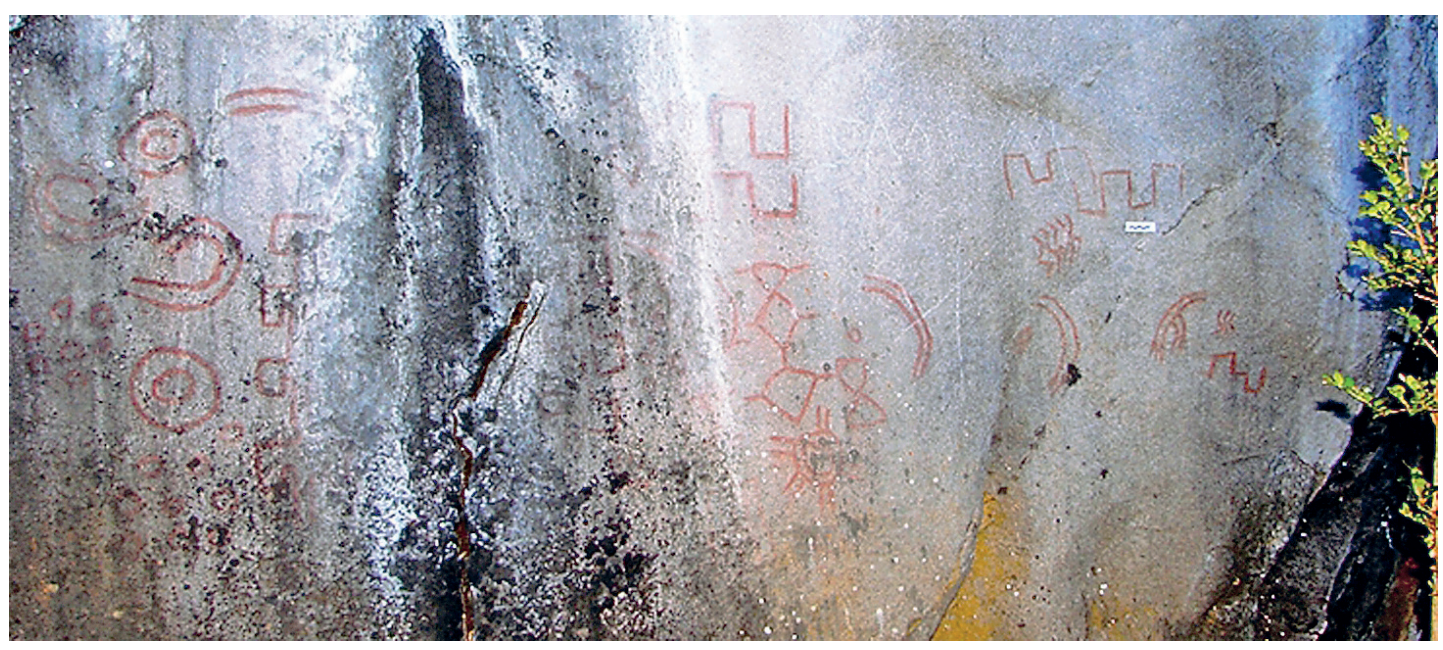

Fig. 6. Panel con pinturas del sitio Torrentoso 01. Chile.

con pinturas antes descrito (ambos son visibles entre sí), a una altura cercana a los $50 \mathrm{~m}$ lineales desde el lecho del río. Las pinturas se disponen sobre una pequeña pared rocosa, de $2 \mathrm{~m}^{2}$ aproximadamente, que se levanta sobre una terraza de origen glacial. Se trata de un único panel con dos motivos compuestos (55 x 43 y 55 x $43 \mathrm{~cm}$, respectivamente) que cubren un área pintada que no supera los 0,5 $\mathrm{m}^{2}$. A diferencia del sitio anterior no presenta daños de origen antrópico (Fig. 7).

A estos dos sitios con pinturas se le agrega un tercero, a cielo abierto y posiblemente habitacional, ubicado en la ribera oeste del Correntoso, inmediatamente adyacente a su cauce (Labarca 2007).

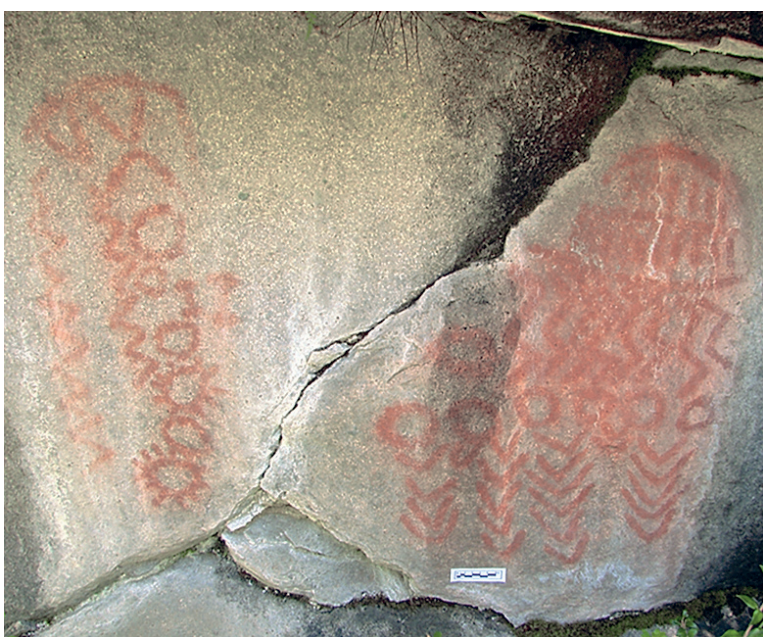

Fig. 7. Motivos del sitio Correntoso 01. Chile.
Debido a que la prospección en el sector chileno se enmarcó dentro de la elaboración de una línea de base arqueológica, para un futuro proyecto de construcción de la ruta que unirá el paso El León con el poblado de Cochamó, los sitios no fueron intervenidos estratigráficamente, por lo que no existe certeza respecto a materialidades asociadas o profundidad cronológica. No obstante lo anterior, las evidentes relaciones estilísticas con los yacimientos del Manso inferior, en territorio argentino, permiten incluirlos dentro de una misma unidad cultural y temporal, relacionada con el estilo de Grecas o TAGC, y dentro de ésta con la Modalidad del Ámbito Boscoso Lacustre del Noroeste de la Patagonia durante el Holoceno tardío.

\section{PALABRAS FINALES}

En líneas generales la información arqueológica, en especial la de arte rupestre, generada en el valle del río Manso inferior (Argentina) y Manso (Chile) es coherente con la proveniente de otros sectores del bosque andino del Norte de Patagonia. Dentro de ésta destacamos la que viene siendo trabajada, y a la cual hicimos referencia, por algunos de los autores de este trabajo en el sector oriental de la cordillera andina: la región del río Epuyén y la de Cholila, ambas en la CA42ํㅜ (Podestá y Tropea 2001; Podestá et al. 2000 y 2007; Tropea 2006) pero también consideramos aquella aportada por colegas que trabajan tanto más al Norte como más al Sur de nuestra área de trabajo. Nos referimos a 
la región del Nahuel Huapi, por un lado y al Parque Nacional Los Alerces, por el otro (Albornoz y Cuneo 2000 y Arrigoni 1997).

Somos concientes de la disímil información proveniente de ambos sectores de la cordillera. Las investigaciones arqueológicas realizadas por más de una década en territorio argentino, contrastan con la ausencia total de trabajos sistemáticos en el sector continental del Occidente andino hacia la misma latitud. Las recientes prospecciones realizadas por uno de nosotros (Labarca 2007) en la cuenca del río Manso, arrojaron la presencia de sitios relacionados estilísticamente con aquellos registrados en el sector argentino del río. Esta nueva evidencia permite incorporar ese sector dentro de la problemática arqueológica regional relacionada con la ocupación de cazadores-recolectores en ambientes boscosos. Al mismo tiempo plantea interesantes preguntas e hipótesis de trabajo referidos a la ocupación humana en ambos sectores cordilleranos y la movilidad de los antiguos habitantes de la región. La ampliación de la información generada hasta ahora permitirá obtener una visión más completa sobre las ocupaciones de estos ambientes boscosos durante el Holoceno tardío.

\section{AGRADECIMIENTOS}

A todos los miembros del equipo de investigación de la Comarca Andina del Paralelo $42^{\circ}$. A los pobladores y amigos del valle del río Manso a ambos lados de la frontera. A las instituciones mencionadas en la Nota 2 y al Ministerio de Turismo de la Provincia de Río Negro, Argentina. A los evaluadores por sus comentarios que nos ayudaron a aclarar algunas ideas y conceptos acá vertidos. A Patricia Solá por sus aportes en temas geográficos. Por último, a la empresa Debar Ltda. que financió el estudio de la línea de base en el Manso (Chile).

\section{BIBLIOGRAFÍA}

ALBORNOZ, A. M. y E. CÚNEO. 2000. Análisis comparativo de sitios con pictografías en ambientes lacustres boscosos de Patagonia Septentrional. En Arte en las Rocas. Arte Rupestre, menhires y piedras de colores en Argentina, M. M. Podestá y M. de Hoyos editoras, pp.163-174. SAA y AINA. Buenos Aires.
ARRIGONI, G. I. 1997. Pintando entre lagos y bosques (las pinturas rupestres del Parque Nacional Los Alerces, Chubut). Revista del Museo de Historia Natural 16 (1/4): 241-268. San Rafael, Mendoza.

ASCHERO, C. A. y A. R. MARTEL. 2003-2005. El arte rupestre de Curuto-5. Antofagasta de la Sierra (Catamarca, Argentina). Cuadernos del INA 20: 47-72. Buenos Aires.

BELARDI, J. B. 2004. Más vueltas que una greca. En Contra viento y marea. Arqueología de Patagonia, M. T. Civalero, P. M. Fernández y A. G. Guráieb compiladores, pp. 591-603. INAPL, SAA. Buenos Aires.

BELlELli, C., M. CARBALLIDO, P. FERNÁNDEZ y V. SCHEINSOHN. 2003. El pasado entre las hojas. Nueva información arqueológica del noroeste de la provincia de Chubut, Argentina. Werken 4: 25-42. Facultad de Ciencias Sociales, Universidad de Chile y LOM Ediciones. Santiago de Chile.

-2007. Investigaciones arqueológicas en el valle del río Manso inferior (Pcia. de Río Negro). Resúmenes ampliados, XVI Congreso Nacional de Arqueología Argentina, Tomo III, pp.309-314. San Salvador de Jujuy: Universidad Nacional de Jujuy.

BELLELLI, C., V. SCHEINSOHN y M. M. PODESTÁ. 2008 Arqueología de pasos cordilleranos: un caso de análisis en la Comarca Andina del Paralelo $42^{\circ}$ y áreas vecinas durante el Holoceno tardío. Aceptado para su publicación al Boletín del Museo Chileno de Arte Precolombino. Santiago de Chile. MS.

CARBALlidO CALATAYUD, M. 2007. Conjuntos líticos del ecotono bosque-estepa en la localidad de Cholila (Provincia del Chubut). En Arqueología Argentina en los Inicios de un Nuevo Siglo. Publicación del XIV Congreso Nacional de Arqueología Argentina, F. Oliva, N. de Grandis y J. Rodríguez compiladores, Tomo I. pp. 309-318. Universidad Nacional de Rosario, Rosario.

INSTITUTO GEOGRÁFICO MILITAR. 1982. Carta topográfica 1:100.000. Hoja 4172-28. Villa Mascardi. Buenos Aires.

GÓMEZ OTERO, J y C. BELLELLI. 2006. Arqueología de la provincia del Chubut. En Patagonia Total. Primera Parte: Historias de la Patagonia. Sociedades y espacios en el tiempo, Susana Bandieri editora, pp. 27-51. Barcelbaires Ediciones y Alfa Centro Literario. Barcelona.

GRADIN, C. 1978. Algunos aspectos del análisis de las manifestaciones rupestres. Revista del Museo Provincial 1: 120-133. Neuquén. 
-1999. Sobre las tendencias del arte rupestre de Patagonia argentina. Segundas Jornadas de Investigadores en Arqueología y Etnohistoria del Centro-Oeste del País, pp. 85-99. Universidad de Río Cuarto, Río Cuarto.

FERNÁNDEZ, P. 2006. Aprovechamiento de recursos faunísticos en los ambientes de estepa y ecotono bosque estepa del norte de la provincia del Chubut. Tesis doctoral, Facultad de Filosofía y Letras, Universidad de Buenos Aires.

-2008. Taphonomy and zooarchaeology in the Neotropics: A view from northwestern Patagonian forest and steppe. Quaternary International 180 (1): 63-74. International Union for Quaternary Research.

HELWIG, K. 2004. Analysis of samples from rock painting sites: Lanfré (Río Negro) and Cerro Pintado (Chubut). Analytical Research Laboratory. Canadian Conservation Institute, Ottawa, Canadá. MS.

LABARCA E., R. 2007. Informe de prospección arqueológica proyecto vial río Manso (Comuna de Cochamó, Región de los Lagos, Chile). Preparado para Debar Ltda. Proyecto Vial Río Manso. MS.

MENGHIN, O. F. A. 1957. Estilos de arte rupestre de Patagonia. Acta Praehistorica 1: 57-87. Centro de Estudios Prehistóricos, Buenos Aires.

PEREYRA, F. X. 2003. Ecoregiones de la Argentina. SEGEMAR, Servicio Geológico Minero. Buenos Aires.

PEREYRA, F. X., C. BELLELLI y M. M. PODESTÁ. 2003. Geoarqueología y preservación de sitios con arte rupestre en los Andes Patagónicos (Provincias de Río Negro y Chubut, Argentina). En CDRom de las Actas del IX Congreso da Associaçao Brasilera de Estudos do Quaternario y II Congreso do Quaternario de Paises de Linguas Ibéricas. 5 pp. Recife, Brasil.

PODESTÁ, M. M. y E. TROPEA. 2001. Expresiones del arte rupestre tardío en el ecotono bosque-estepa (Comarca Andina del Paralelo 42․ Patagonia. Presentado al XVI Congreso Nacional de Arqueología Argentina. Universidad Nacional de Rosario. En prensa. Rosario.

PODESTÁ, M. M. y A. ALBORNOZ. 2007. El arte rupestre del sitio Paredón Lanfré dentro del contexto arqueológico del valle del río Manso inferior (Pcia. de Río Negro). Resúmenes ampliados, XVI Congreso Nacional de Arqueología Argentina, Tomo III, pp.429-434. San Salvador de Jujuy: Universidad Nacional de Jujuy.
PODESTÁ, M. M., R. S. PAUNERO y D. S. ROLANDI. 2005. Arte Rupestre de Argentina Indígena. Patagonia. Academia Nacional de la Historia. GAC. Buenos Aires.

PODESTÁ, M. M., C. BELLELLI, P. FERNÁNDEZ, M. CARBALLIDO y M. PANIQUELLI. 2000. Arte rupestre de la Comarca Andina del Paralelo 42: un caso de análisis regional para el manejo de recursos culturales. En Arte en las Rocas. Arte rupestre, menhires y piedras de colores en Argentina, editado por M. M. Podestá y M. de Hoyos, pp. 175-201. Sociedad Argentina de Antropología y Asociación Amigos del INAPL. Buenos Aires.

PODESTÁ, M., M., C. BELLELLI, V. SCHEINSOHN, P. FERNÁNDEZ, M. CARBALLIDO CALATAYUD, A. FORLANO, P. MARCHIONE, E. TROPEA, A. VASINI, J. ALBERTI, M. GALLO y G. MOSCOVICI VERNIERI. 2007. Arqueología del valle del río Epuyén (El Hoyo, Chubut, Patagonia Argentina). En Arqueología de Fuego-Patagonia. Levantando piedras, desenterrando huesos...y develando arcanos. Editado por Flavia Morello, Mateo Martinic, Alfredo Prieto y Gabriel Bahamonde, pp. 427-442. Punta Arenas.

PODESTÁ, M. M., A. M. ALBORNOZ, A. VASINI y E. TROPEA. 2008. Peumayén 2: un sitio con arte rupestre en el valle del río Manso inferior (Provincia de Río Negro, Argentina. Ponencia presentada a las VII Jornadas de Arqueología de Patagonia. 21 a 25 de abril de 2008. Ushuaia.

SCHEINSOHN, V. y S. MATEUCCI. 2004. Spaces and species: archaeology, landscape ecology and spatial models in northern Patagonia. Before Farming [online] 2004/1 article 2, http://www.waspress.co.uk/journals/beforefarming/journal_20041/abstracts/index.php.

-2005. A regional model of archaeological distributions for Comarca Andina del Paralelo 42 , Patagonia, Argentina. En Uso de Sistemas de Información Geográfica (SIG) en la arqueología sudamericana, editado por María José Figuerero Torres y A. Izeta, BAR International Series, Oxford. En prensa.

TROPEA, E. 2006. Expresiones artísticas tardías en el ecotono bosque-estepa. El caso de cuatro sitios con arte rupestre en la localidad de Cholila (Comarca Andina del Paralelo $42^{\circ}$, Patagonia argentina). Tesis de Grado. Carrera de Ciencias Antropológicas. Facultad de Filosofía y Letras. Universidad de Buenos Aires, Buenos Aires. MS. 
